\title{
Performance Analysis of Node-Disjoint Multipath in MANET: A Modified Approach
}

\author{
Rahma Bintey Mufiz Mukta \\ Dept. of Computer Science \& Engineering \\ Chittagong University of Engineering \& Technology \\ Chittagong, Bangladesh
}

\begin{abstract}
MANET provides a good platform for any time anywhere networking. Nodes in MANET communicate via wireless multi hop links. Due to frequent node movement routing algorithm in wired network is not suitable for MANET. AODV is the most popular routing algorithm. Route maintenance is the major issue in AODV when there is only one path is established between source and destination. Wireless link breakage is higher in ad-hoc networks due to the node movement, so alternative paths are needed for the route maintenance. This paper gives a new idea to discover multiple node-disjoint routing paths. This extended AODV balances energy and traffic load on whole network to increase the network lifetime. Simulation results show that the performance of proposed Maximum Multipath AODV (MM-AODV) is much better than that of existing AODV.
\end{abstract}

\section{General Terms}

AODV, Routing algorithm, Wireless communication

\section{Keywords}

MANET; AODV; Multipath Routing Protocol; Node Disjoint Multipath; NS2

\section{INTRODUCTION}

A mobile ad hoc network is a collection of wireless mobile nodes that dynamically establishes the network in the absence of fixed infrastructure [1]. One of the distinctive features of MANET is, each node must be able to act as a router to find out the optimal path to forward a packet. As nodes may be mobile, entering and leaving the network, the topology of the network will change continuously. MANETs provide an emerging technology for civilian and military applications. One of the important research areas in MANET is establishing and maintaining the ad hoc network through the use of routing protocols. Well known source-initiated on demand routing protocols include AODV and DSR [2]. These protocols are based on the strategy of only finding valid routes once they are needed by the source node. This procedure, known as route discovery involves the route request phase (RREQ) and the route reply phase (RREP). All of these protocols construct a single-path route between a source node and a destination node. Whenever communication link breaks on the active route, each protocol has to invoke a route discovery process. Delay in route discovery and frequent route discovery process can cause the performance adversely. The multipath on-demand protocols are helpful to lighten the problem by finding multiple paths in a single route discovery process. The new route discovery is needed only when all routes to the destination fail or when there only remains a single path available. The main focus is not choosing multi-path or single path, but how to discover maximum possible complete node-disjoint paths. Discovery of complete node-disjoint multipath has been proposed in
[3], [4], [5] and [6]. In AODV [7] the nodes respond at most once to RREQ, so that it can reduce the total number of RREP messages. But the total number of RREQ messages is still higher. In [8] NDAMR (Node-Disjoint Alternative Multiple Path) and in [9] DPNR (Dual Path Node-disjoint Routing) are two efficient processes for data salvation in case of link failure. These routing protocols maintain only two shortest backup paths in the source and destination nodes by combining the AODV-BR strategy and on-demand node-disjoint multi-path routing protocols. Most of ondemand multipath routing protocols such as AOMDV [10] and [3] use a simple method of multipath discovery, where source initiates route request whenever needs to send data and destination node sends reply for each received route request even it is from same source. AOMDV introduces an idea of hop count and finds link disjoint paths. However, in several cases multi path discovery fails and discover much fewer number of paths than currently available. Another method of multipath discovery is source routing. Protocols such as [4] and [11] use this method. The method discovers multi-paths on demand recording router address in RREQ and destination node decides to select paths. This method may seem very simple, but it causes a lot of overhead and routing load due to heavy RREQ. In [12], the paper proposed a new idea for finding an alternative path in AODV during link failure. In this scheme the intermediate nodes plays an important role to avoid needless route discovery process. Though this ensures the performance enhancement by reducing the number of route discovery process, but an intermediate node has to cache more route information when a RREP message sent. This increases message size each time. Although these protocols build multiple paths on demand, most of them could not guarantee to find all available complete disjoint paths even though they use much complex methods. In this paper, a novel method of discovering maximum available complete node-disjoint multiple paths in the network is proposed. The method modifies and extends AODV to enable path discovery and accumulation feature.

The main motivation of this paper is to provide multiple routes in network communications, particularly in MANETs.

The rest of the paper is organized as follows: Section II gives a brief introduction of AODV routing protocol. Section III gives an overview of modified AODV (MMAODV) routing protocol. Section IV describes the performance evaluation metrics. Section V gives the Simulation setup, results and performance comparison of the two routing protocols. Section VI concludes the paper.

\section{AD-HOC ON DEMAND DISTANCE VECTOR ROUTING (AODV)}

Ad-hoc on demand Distance Vector Routing (AODV) is a reactive routing algorithm, improvement over DSDV routing 
protocol algorithm. It minimizes the number of broadcasts by creating routes on-demand as opposed to all possible routes as in DSDV. AODV is a loop- free, single path, distance vector protocol based on hop-by-hop routing approach. There are two main procedures in AODV:

1. Route discovery

\section{Route maintenance}

\subsection{Route Discovery}

When a source node desires to send a message to some destination node, and doesn't have a valid route to the destination, it initiates a path discovery process to locate the other node. It broadcasts a route request (RREQ) control packet to its neighbors, which then forward the request to their neighbors, and so on, either the destination or an intermediate node with a new route to the destination is located. RREQ packet contains:

\section{Source address \\ Source Sequence Number \\ Broadcast Id \\ Destination Address \\ Destination Sequence Number \\ Hop Count}

The AODV protocol utilizes destination sequence numbers to ensure that all routes contain the most recent route information. Each node maintains its own sequence number. During the forwarding process the RREQ intermediate nodes record the address of the neighbor from which the first copy of the broadcast packet is received in their route tables, thereby establishing a reverse path. Once the RREQ reaches the destination or an intermediate node with a fresh enough route, the destination or the intermediate node responds by unicasting a route reply (RREP) control packet back to the neighbor from which first received the RREQ $[13,14]$. Here RREP packet contains:

\section{Source address \\ Destination Address \\ Destination Sequence Number \\ Hop Count \\ Life Time}

\subsection{Route Maintenance}

A route discovered between a source node and destination node is maintained as long as needed by the source node. The destination node or some intermediate node moves, the node upstream of the break initiates Route Error (RERR) message to the affected active upstream neighbors/nodes. Consequently, these nodes propagate the RERR to their predecessor nodes. This process continues until the source node is reached. When RERR is received by the source node, it can either stop sending the data or reinitiate the route discovery mechanism by sending a new RREQ message if the route is still required[15,16]. AODV also supports periodic HELLO messages to ensure symmetric links, as well as to detect link failures. A node can keeps track of its Neighbors by getting a HELLO message that each node broadcast at set intervals.

\section{PROPOSED METHOD}

The goal of this proposed method is to discover maximum available complete node-disjoint paths from a source to a destination depending on the number of neighbors of source node. Analyzing previous works, we become known that number of multi-paths between source and destination depends mostly on two parameters: neighbors of source node and neighbors of destination node. Number of node-disjoint multi-paths is not more than number of neighbors of source and destination. The term 'disjoint path' is used to define how many nodes participate more than twice in discovery of multiple paths from a source to a destination. And the nodedisjoint multipath means each node participates in only and only one path.

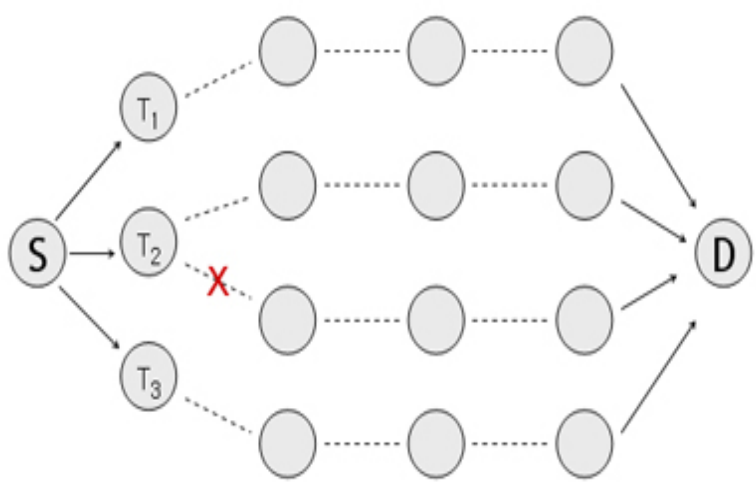

Fig 1: Maximum Multipath

As shown in figure 1 , even though there are enough intermediate nodes to build more multipath, complete nodedisjoint multi-paths can be only 3 - neighbors of source node. Therefore deciding multi-paths according to neighbors of source and destination would be exact and efficient.
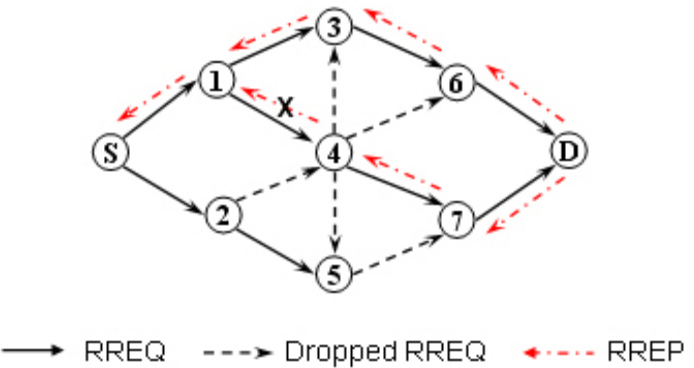

Fig 2: Multipath discovery failure

Again, in several cases node-disjoint multipath discovery fails and discover much fewer number of paths than currently available. Figure 2 shows one example for those cases. When source node broadcast route request (RREQ) node 1 and 2 receives first and pass on next nodes. Some nodes like 4, 5, 6, and 7 may receive redundant RREQ and they discard it (shown in figure as dashed arrow). When destination node receives RREQ from node 6 and 7, the node sends RREP through reverse path. First RREP arrives to source node through D-6-3-1-S and second RREP is discarded on node 1 , because node 1 already received RREP. As result, source node can build only a single path S-1-3-6$\mathrm{D}$, even though there are other paths. If node 1 does not discard RREP source node could build another complete node disjoint multipath that can be used latter in case of any link breakage through 1-3-6-D. 


\subsection{Route Discovery}

Since the discovery method is reactive routing protocol, no permanent routes is stored in nodes. The source node initiates route discovery procedure by broadcasting the ROUTE REQUEST (RREQ) message. When neighbor nodes of source (one-hop nodes) receive RREQ, the nodes, we call them secondary sources; include their address to the message. RREQ packet contains:

\section{Source address}

Source sequence number

Broadcast id

Destination address

Destination sequence number

\section{Secondary Source Address}

Hop count

All intermediate nodes uniquely identify redundant messages using source address and sequence number. Sequence number is set in source node and incremented when source node initiates route request. Neighbor nodes of destination allow two duplicate messages and if secondary source is different. Destination node generates ROUTE REPLY (RREP) for each secondary source. Secondary sources with multiple route replies, first one will be used and second one will be stored. When the first link fails, the node sends a RERR message to the source node. The source node can select the next available route entry from its table. The proposed scheme says, instead of simply forwarding a route error message, the intermediate node can notify the source node by adding next hop and hop count, if it possesses another valid route to the destination. If the first link of T2-D fails, a RERR message is used to notify other nodes that the loss of that link has occurred. When the source node getting a RERR message it can check any valid route is available through the same intermediate node $\mathrm{T} 2$.

\subsection{Conditions}

Each path from the neighbors of destination:

1. If two secondary source paths share a path, only one path is selected.

2. When two paths overlap with same secondary source, first one will be selected and second one will be stored;

3. If neighbor nodes of destination are fewer than secondary sources, number of selected paths is not more than number of destination neighbors;

4. If all available paths are fewer than neighbors of source and/or destination due to intermediate nodes, number of selected paths can be less than source neighbors or destination neighbors.

Each route reply builds a single path to each secondary source and source node can build multipath with it. Goal of our proposed method is to build maximum complete nodedisjoint multipath as shown in figure 3 where other than the source node $\mathrm{S}$ and destination node $\mathrm{D}$, paths have no node in common. If intermediate nodes receive RREP several times due to secondary source address, the nodes just discard it.

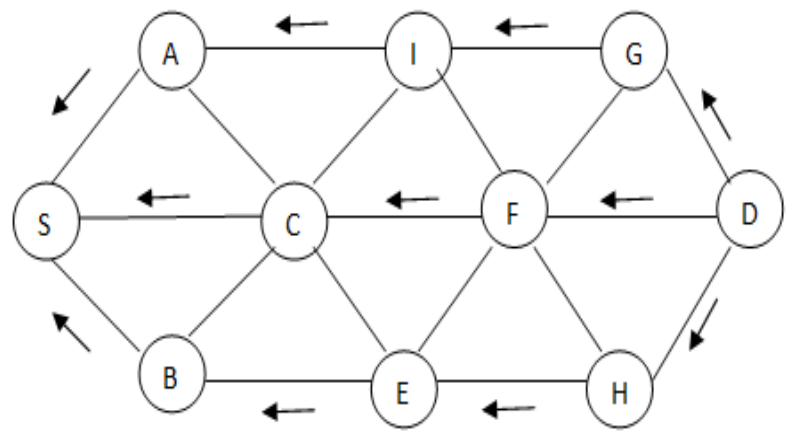

Fig 3: Propagation of RREP through node-disjoint paths

After discovery of multipath, they can be used for several ways. Hop one by one or use exhaustively. Hopping has different advantages mentioned in [17] such as security, traffic load and energy distribution. Exhaustively using may increase packet delivery ratio and decrease packet overhead.

\section{PERFORMANCE EVALUATION METRICS}

To evaluate performance of MM-AODV with that of AODV protocol, we compare them using five metrics:

\subsection{Average Packet Delivery Ratio}

Packet delivery ratio is the fraction of packets sent by the application that are received by the receivers and is calculated by dividing the number of packets received by the destination through the number of packets originated by the application layer of the source. Hence, the greater value of packet delivery ratio means better performance of the protocol. Following is the equation to calculate the packet delivery ratio.

\section{$P D R=\Sigma$ No of packet receive $/ \Sigma$ No of packet send}

\subsection{Average Energy Consumption}

Mean value of energy consumption in each node. Hence, the lower value of average energy consumption means better performance of the protocol.

Average Energy Consumption $=\Sigma$ Energy consumption $/ \Sigma$ No of nodes

\subsection{Normalized Routing Load}

The normalized routing load defines the number of routing packets transmitted per data packet delivered at the destination node. Each hop-wise transmission of a routing packet is counted as one transmission. It is given by.

Normalized routing load $=\Sigma$ routing packets $\Sigma$ received data packets

\subsection{Average Throughput}

It is defined as the total amount of data per time unit that is delivered from one node to another via a communication link. Or it is the number of packets received by all the nodes in the network. Hence, the greater value of average throughput means better performance of the protocol. It can be calculated by the following equation:

Throughput $=\Sigma$ RecvdSize $/ \Sigma($ stopTime-startTime $)$

\subsection{Average End to End Delay}

This includes all possible delays caused by buffering during route discovery latency, queuing at the interface queue, retransmission delays at the MAC, and propagation and transfer times. It should be less for high performance. 


\section{End to end delay $=\Sigma($ arrive time - send time $)$}

The end-to-end-delay is averaged over all surviving data packets from the sources to the destinations.

\section{SIMULATION \& RESULTS}

Simulations are implemented in Network Simulator (NS-2) [18] from Lawrence Berkeley National Laboratory (LBNL). Distributed Coordination Function (DCF) of IEEE 802.11 for wireless LANs is used as the MAC layer protocol, and Lucent's WaveLAN is used as the radio model. WaveLAN supports a nominal bit rate of $2 \mathrm{Mb} / \mathrm{s}$ and a radio range of $250 \mathrm{~m}$. The simulations are based on constant bit rate (CBR) traffic sources and 64 packet send buffers. The data packet payload is 512 bytes. Packets are dropped if they remain in the send buffer for more than 30 seconds. All packets sent by the routing layer are queued in the interface queue which has a maximum capacity of 50 packets. Routing packets have higher priority than data packets. Mobile nodes were placed into a square area of $1000 \mathrm{~m} \mathrm{X} 1000 \mathrm{~m}$. Mobility model is random way point model (when the node reaches its destination, it pauses for several seconds, e.g., 1s, then randomly chooses another destination point within the field, with a randomly selected constant velocity). Each simulation ran for 200 seconds. Each data point on the simulation curves in the figures represents a mean of five protocol runs with identical traffic/mobility scenarios. Simulation is conducted based on node density and node speed. AODV and proposed MM-AODV are compared in this simulation. Figure 4 is the simple simulation model of AODV and figure 5 is the simple simulation model of proposed protocol, Maximum Multipath AODV (MM-AODV). Here number of nodes is varied among 10, 20, 30, 50 and 75 and mobile speed is uniformly distributed between 0 and MAXSPEED (we choose MAXSPEED $=2,5,10,15,20 \mathrm{~m} / \mathrm{s}$ respectively).

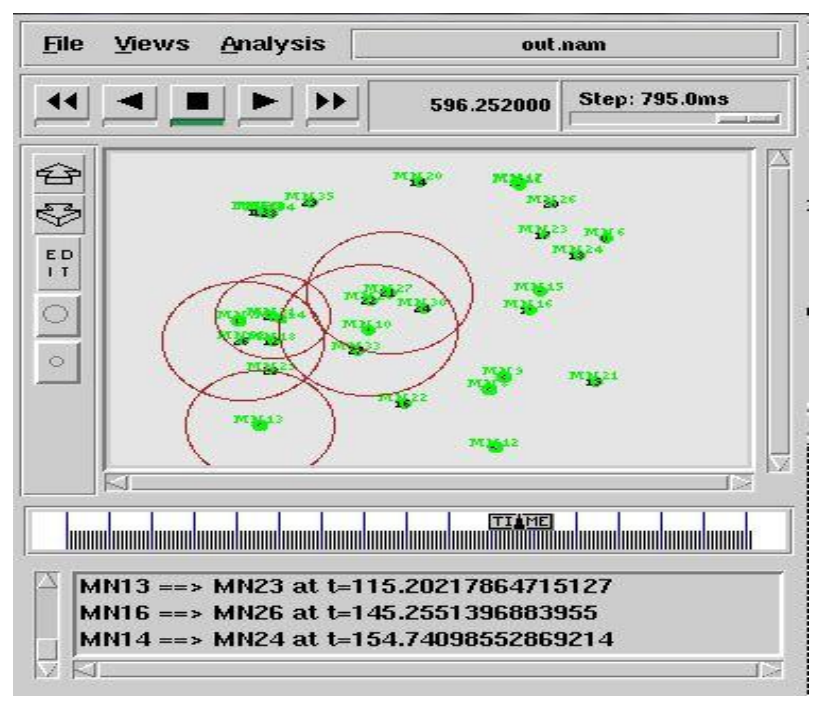

Fig 4: Simulation Model of AODV

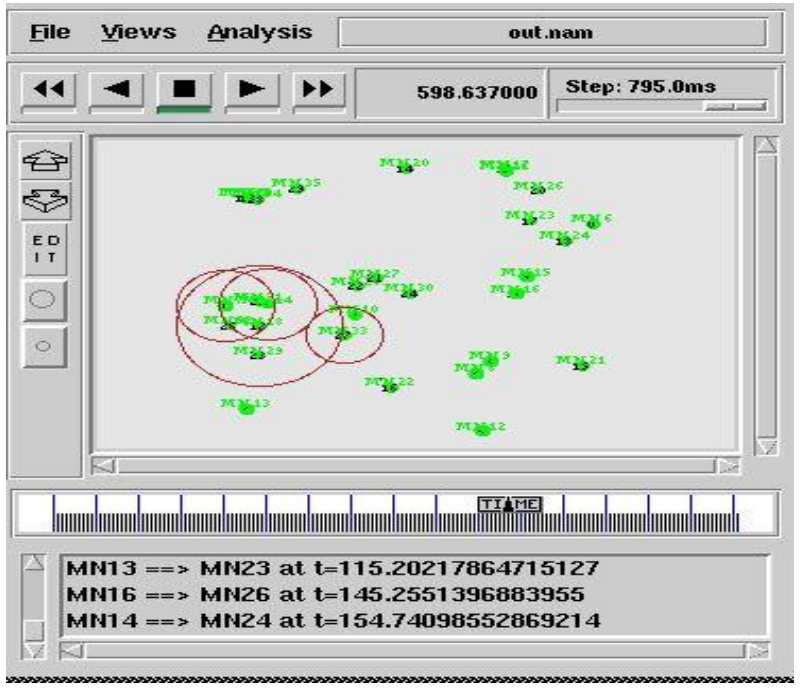

Fig 5: Simulation Model of MM-AODV

\subsection{Based on Node Density}

The number of nodes per group varies as 10,20,30,50 and 75 respectively.

Figure 6 shows packet delivery ratio of each protocol when number of nodes varies. When there are more nodes in network MM-AODV can show better performance because more multipath are available.

Figure 7 shows the average energy consumption of each protocol when number of nodes varies. This is to mention that average energy consumption is a mean value of consumed energy of each node at the end of simulation. MM-AODV maintains lower energy consumption because it floods less route requests. For small number of nodes the performance of two routing protocols is same. But with the increasing number of nodes energy consumption of MMAODV reduces gradually. It is of $12 \%$ lower than AODV for 75 nodes.

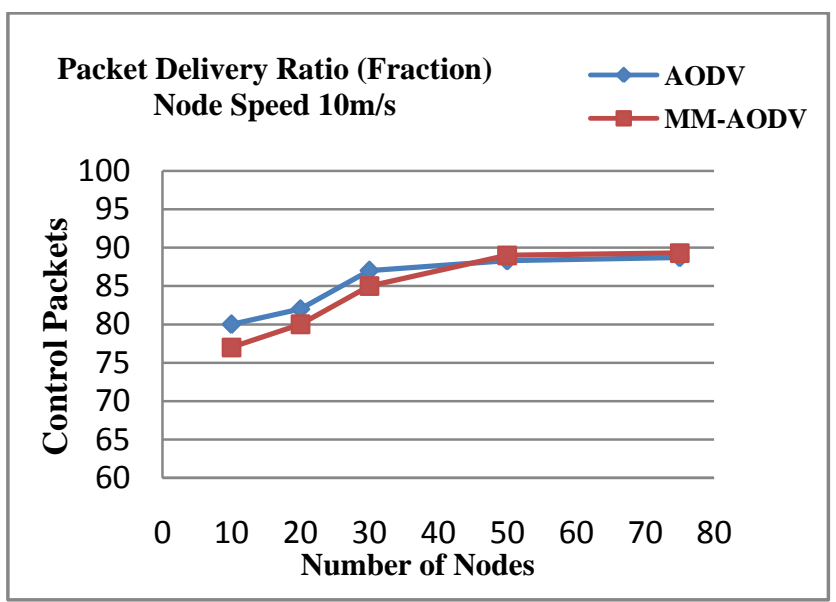

Fig 6: Packet delivery Ratio, when number of Nodes varies 


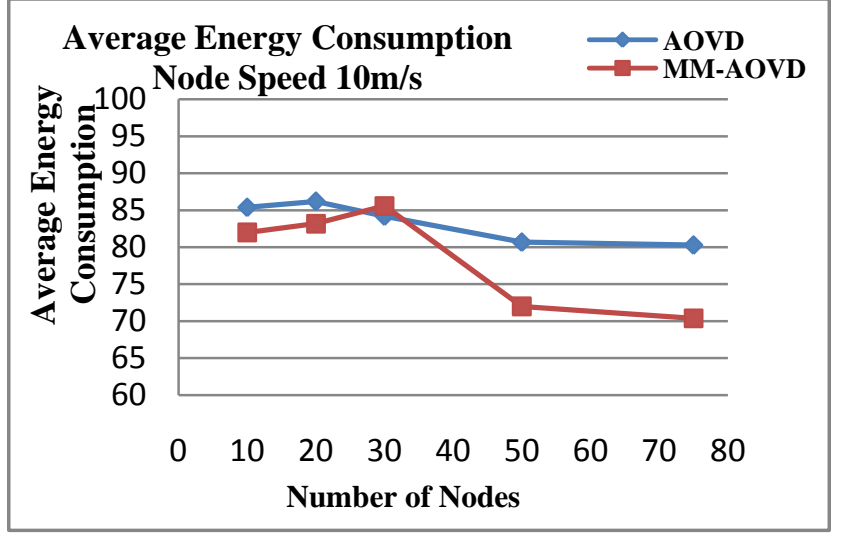

Fig 7: Average Energy Consumption, when number of nodes varies

Figure 8 shows the routing overhead of each protocol according to the variation of number of nodes. The normalized routing load (NRL) of these two compared protocols is almost same for small number of nodes. NRL of each protocol increases in proportion to the increase of node density. Because more requests are flooded over network, yet MM-AODV shows better performance in it.

Figure 9 shows average throughput of each protocol, when the number of nodes varies. As we can see from the figure, the throughput is high for Proposed Protocol MM-AODV. For small number of nodes average throughput of two compared protocols is almost same. When number of nodes increases, average throughput rises. MM-AODV shows $23 \%$ increase in average throughput than AODV for 75 nodes.

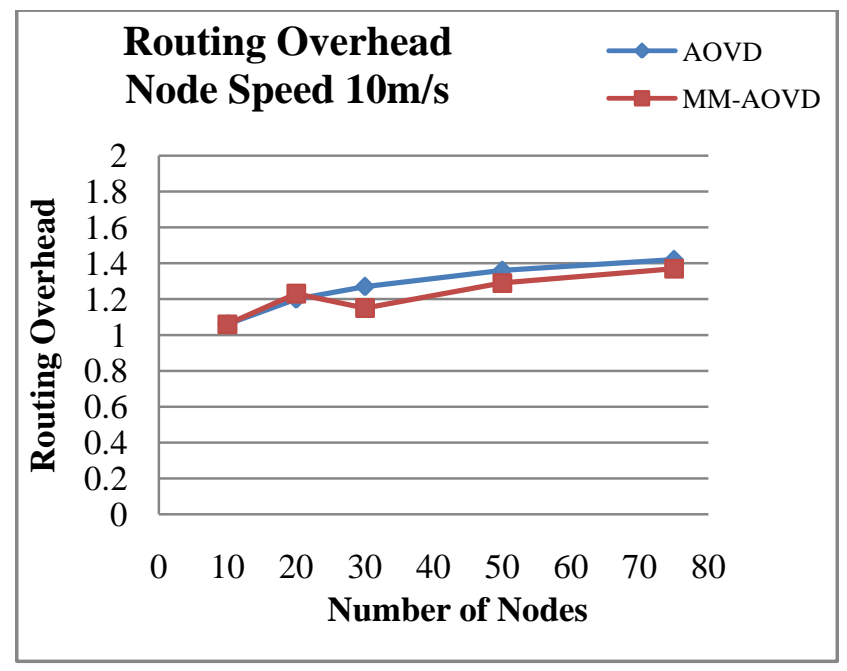

Fig 8: Routing Overhead, when number of nodes varies

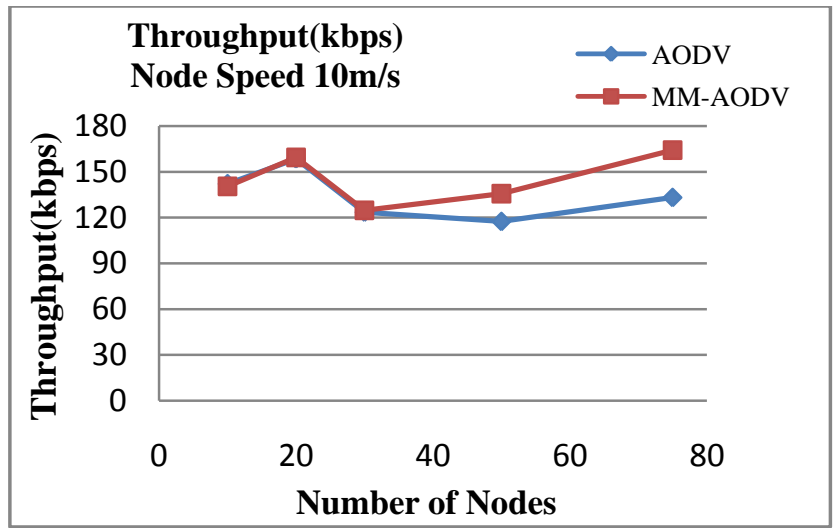

Fig 9: Average Throughput (Kbps), when number of nodes varies

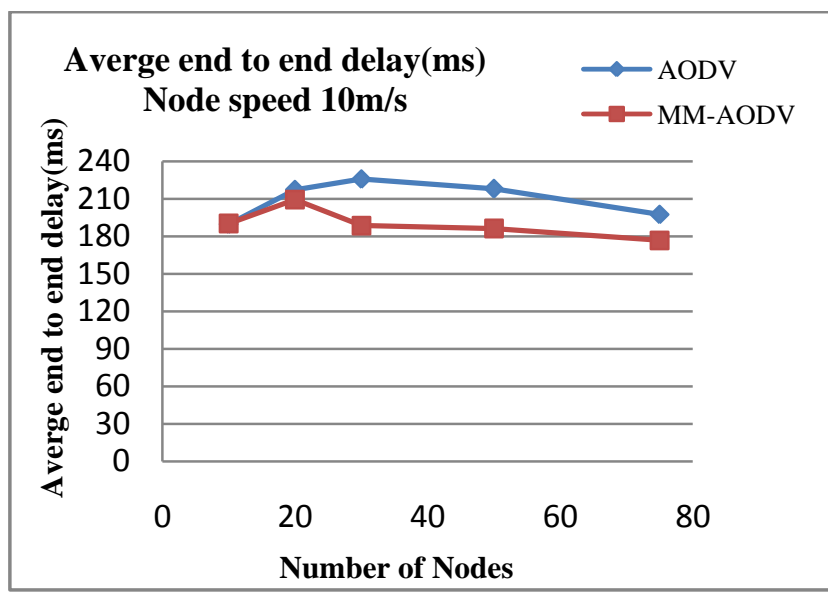

Fig 10: Average end to end delay, when number of nodes varies

Figure 10 shows the average end-to-end delay occurred for the AODV and MM-AODV. As we can see from the figure, the delay is less for proposed protocol MM-AODV, because of more multipath. For 30 nodes the delay is almost $16 \%$ lower for MM-AODV than AODV. Although, the delay increases with increasing number of nodes, but MM-AODV shows better performance in it.

\subsection{Based on Node Speed}

The node speed is uniformly distributed between 0 and MAXSPEED (we choose MAXSPEED $=2,5,10,15,20$ $\mathrm{m} / \mathrm{s}$ respectively).

Figure 11 shows packet deliver ratio (PDR) of each protocol when node speed varies. Initially MM-AODV shows better PDR than AODV. When node speed increases path break probability also increases, therefore packet delivery rate of MM-AODV become slightly less than AODV.

Figure 12 shows the average energy consumption of each protocol when node speed varies. This is to mention that average energy consumption is a mean value of consumed energy of each node at the end of simulation. MM-AODV maintains lower energy consumption because it floods less route requests. When node speed increases, average energy consumption reduces for MM-AODV. 


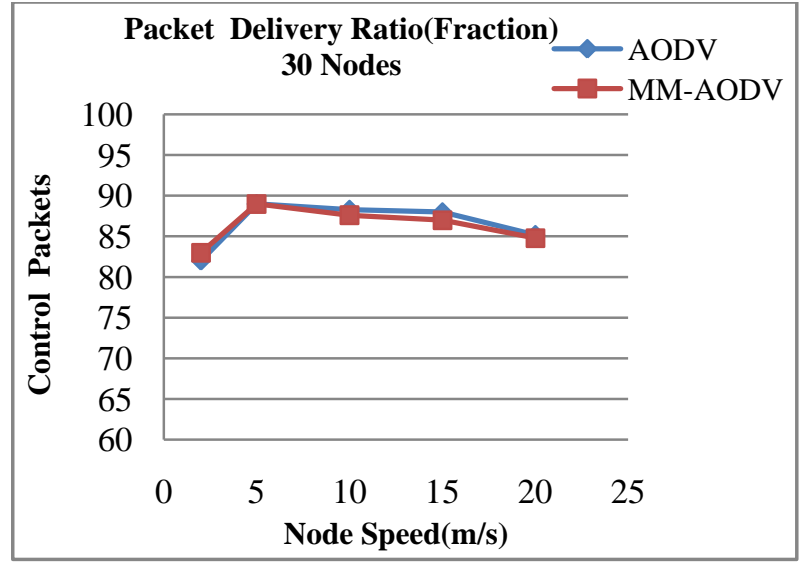

Fig 11: Packet Delivery Ration, when node speed varies

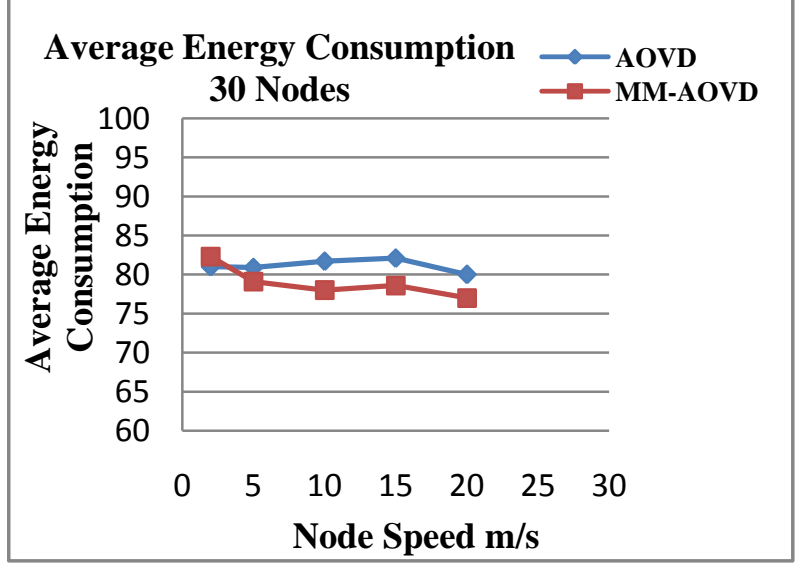

Fig 12: Average Energy Consumption, when node speed varies

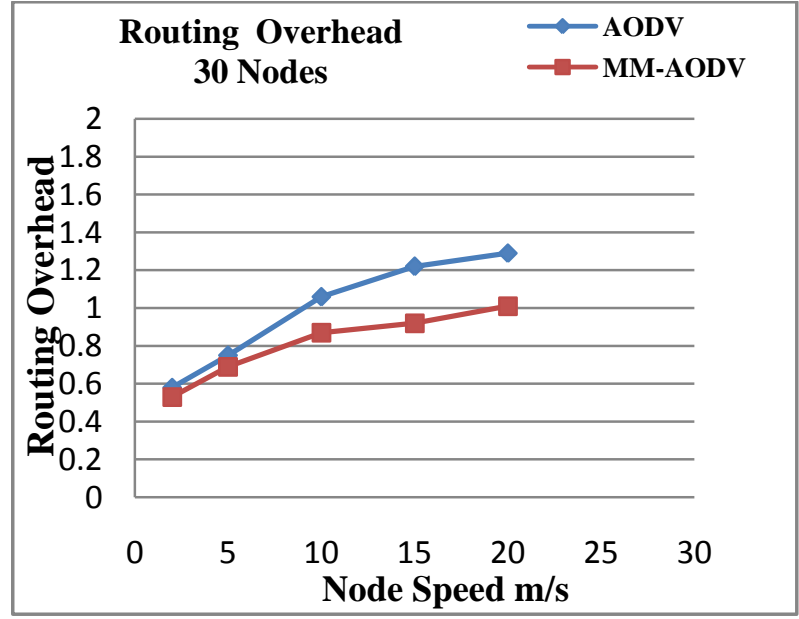

Fig 13: Routing overhead, when node speed varies

Figure 13 shows the routing overhead of each protocol according to the variation of speed. Normalized routing load of each protocol increases in proportion to the increase of speed. Because more requests are flooded over network, yet MM-AODV shows better performance in it. Initially the performance of two compared protocols is almost same, but with increasing node speed of $15 \mathrm{~m} / \mathrm{s}$, NRL reduces to almost $25 \%$ lower than AODV. This percentage is of $22 \%$ lower than AODV when the node speed is $20 \mathrm{~m} / \mathrm{s}$.
Figure 14 shows the average throughput of each protocol when node speed varies. From the figure, the throughput is high for proposed protocol MM-AODV, when compared to AODV. When node speed increases, the average throughput also increases.

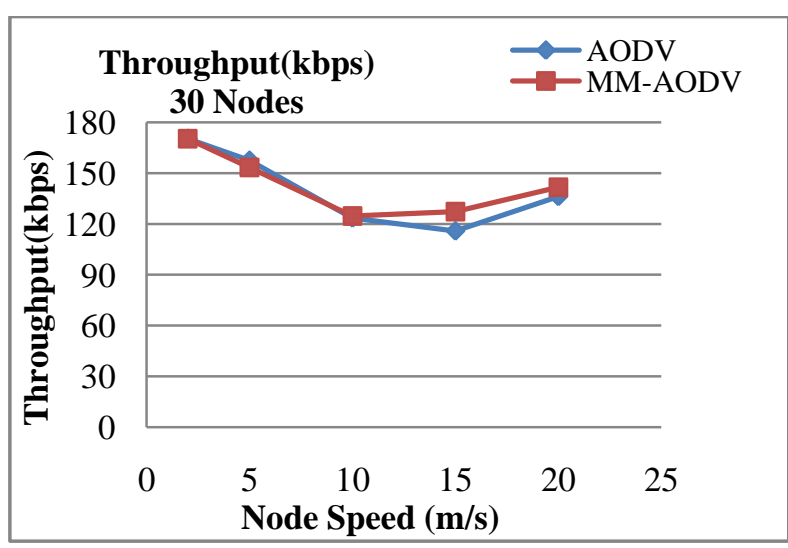

Fig 14: Average Throughput (Kbps), when node speed varies

Figure 15 shows the average end-to-end delay occurred for the AODV and MM-AODV. From the figure, the delay is less for proposed protocol MM-AODV, because of more multipath. End to end delay of MM-AODV is $16 \%$ lower than AODV for $10 \mathrm{~m} / \mathrm{s}$ node speed. For the node speed of $20 \mathrm{~m} / \mathrm{s}$, delay is almost $21 \%$ lower for MM-AODV. Therefore, average end to end delay gradually decreases with the increase of node speed.

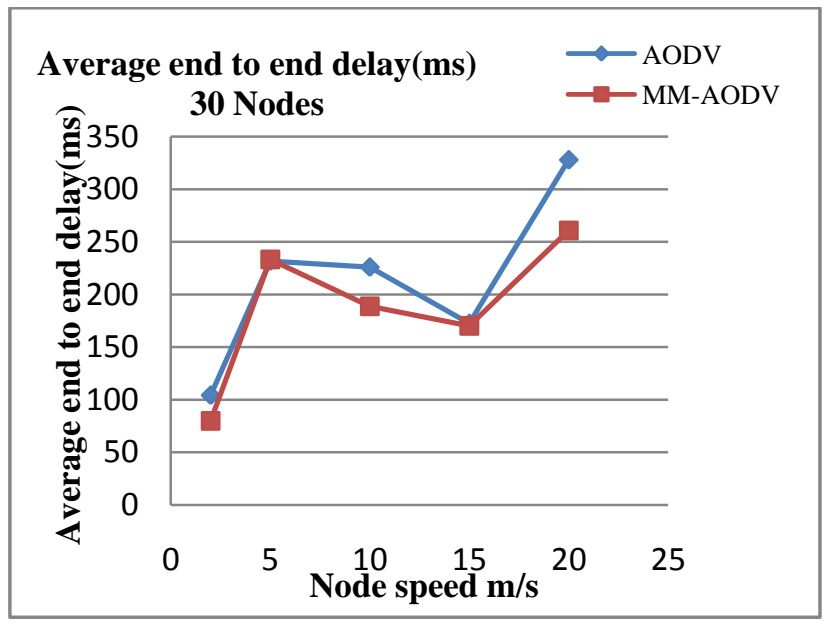

Fig 15: Average end to end delay, when node speed varies

\section{CONCLUSION}

AODV keeps on using the same path. Exhaustively using a single path increases traffic load and consumes faster the energy of nodes on the path. The multipath routing addresses this problem by providing more than one route to a destination node. This multipath routing effectively reduces the frequency of route discovery, therefore the latency for discovering another route is reduced when currently used route is broken. This paper presents a method Maximum Multipath AODV (MM-AODV) for finding complete nodedisjoint paths between a pair of nodes in an on demand manner. Without commences a new route discovery process, the new route can be set up and the active transmission can be continued. This approach does not have any additional 
computational complexity and this uses the same principle of AODV protocol which is universally accepted. MM-AODV supports stability i.e. it increases mean time to failure of the nodes by distributing the burden of routing. The results of comparing two routing protocols, Adhoc on demand distance vector (AODV) and proposed Maximum Multipath AODV (MM-AODV), show that the proposed protocol has better delivery ratio with less energy consumption and routing overhead when compared with the existing network protocol. Therefore, multipath routing appears to be a promising technique for ad hoc routing protocols. In future there is the scope of extending this method as cooperative for making it more energy efficient.

\section{REFERENCES}

[1] C.Sivaram murthy, B.S.Manoj, "Adhoc wireless networks: Architectures, and protocols", Pearson Education, 2004

[2] S. A. Ade \& P. A. Tijare, "Performance comparison of $A O D V, D S D V, O L S R$ and DSR routing protocols in mobile ad-hoc networks", International Journal of Information Technology and Knowledge management, July-December 2010, Vol. 2, No. 2,pp. 545-548.

[3] Zhi Li and Yu-Kwong Kwok, "A new multipath routing approach to enhancing TCP Security in ad hoc wireless networks", Proceedings of the 2005 International Conference on Parallel Processing Workshops (ICPPW'05), pp. 372-379, June 2005

[4] Xuefei Li and Laurie Cuthbert, "Stable node-disjoint multipath routing with low overhead in mobile ad hoc networks", In Proceedings of the IEEE Computer Society's 12th Annual International Symposium on Modeling, Analysis, and Simulation of Computer and Telecommunications Systems, MASCOTS '04, pp. 184-191, 2004.

[5] M. K. Marina and S. R. Das "On-demand multi path distance vector routing in ad hoc networks," Proceedings of ICNP 2001, pp. 14-23, Nov. 2001.

[6] Nasipuri and S. R. Das, "On-demand multipath routing for mobile ad hoc networks," Proceedings of IEEE ICCCN'99, Boston, MA, Oct. 1999, pp. 64-70.

[7] E. M. Royer, C. K. Toh, "A review of current routing protocols for ad hoc mobile wireless networks", IEEE Personal Communications Magazine, Vol. 6, No. 2, April 1999, pp. 46-55.

[8] Chu-Hsing Lin, Fuu-Cheng Jiang, Jen-Chieh Chang, "Node-disjoint alternative dual-path routing for data salvation in mobile ad hoc networks", Ninth International Conference on Parallel and Distributed Computing, Applications and Technologies, (PDCAT '08), Dunedin, Otago, New Zealand, pp. 342-349, Dec. 2008.

[9] Fuu-Cheng Jiang, Chu-Hsing Lin, Der-Chen Huang, Chao-Tung Yang, "Dual paths node-disjoint routing for data salvation in mobile ad hoc", The Journal of Supercomputing, Springer Netherlands, Vol. 59,Issue 1,pp. 268-296, January 2012

[10] M. K. Marina and S. R. Das "On-demand multi path distance vector routing in ad hoc networks," Proceedings of ICNP 2001, pp. 14-23, Nov. 2001.

[11] S. J. Lee and M. Gerla, "Split multipath routing with maximally disjoint paths in ad hoc networks," Proceedings of ICC 2001, vol. 10, pp. 3201-3205, June 2001

[12] Priya K G et al., "A novel approach to find the alternative path for route maintenance in AODV", International Journal of Computer Science \& Engineering Technology (IJCSET), July 2013, Vol. 4, No.7, pp. 1065-1069, 2013.

[13] P. Bose, P. Morin, I. Stojmenovic and J. Urrutia, "Routing with guaranteed delivery in ad hoc wireless networks", ACM DIALM 1999, 48- 5; ACM/Kluwer Wireless Networks, 7, 6, 609-616, November-2001.

[14] L Xue, M S Leeson and R J Green, "Internet connection protocol for ad hoc wireless networks", Communications \& Signal Processing Group,

[15] Srdjan Krco and Marina Dupcinov, "Improved neighbor detection algorithm for AODV routing protocol", IEEE Communications Letters, vol. 7, No.12, December 2003.

[16] Venugopalan Ramasubramanian and Daniel Mossee "BRA: A bidirectional routing abstraction for asymmetric mobile ad hoc networks", IEEE/ACM Transactions on Networking, Vol 16, No.1,February 2008.

[17] Elmurod Talipov, Donxue Jin, Jaeyoun Jung, Ilkyu Ha, Youngjun Choi, and Chonggun Kim,"Path hopping based on Reverse AODV for security" APNOMS2006, LNCS 4283, pp. 574-577, 2006.

[18] Last date of access (March 24, 2013). [Online]. Available: http://www.isi.edu/nsnam/ns. 